\title{
THE GENERALIZATION OF A LEMMA OF M. S. KAKEYA
}

\section{J. GERONIMUS}

We shall prove the following:

Lemma. It is always possible to find the unique polynomial

$$
\phi^{*}(z)=\sum_{k=0}^{2 s} \gamma_{k}^{*} z^{k}
$$

of degree $2 s$ possessing the following properties:

I.

$$
\phi^{*}(z)=c i^{2}(z) \tau(z) \tau^{*}(z), \quad c=\text { const., }
$$

the polynomial $i(z)$ of degree $\sigma \leqq s$ having all roots in the domain $|z|>1$ :

$$
i(z)=\prod_{i=1}^{\sigma}\left(z-a_{i}\right), \quad\left|a_{i}\right|>1, \quad i=1,2, \cdots, \sigma,
$$

and the polynomial $\tau(z)$ being of degree $\nu=s-\sigma$ :

$$
\tau(z)=\prod_{i=1}^{\nu}\left(z-\alpha_{i}\right), \quad \tau^{*}(z)=z^{\nu} \bar{\tau}\left(\frac{1}{z}\right)=\prod_{i=1}^{\nu}\left(1-z \bar{\alpha}_{i}\right) .
$$

II. It is subject to the conditions

$$
\omega_{i}\left(\phi^{*}\right)=\sum_{k=0}^{2 s} \gamma_{k}^{*} c_{k}^{(i)}=d_{i}, \quad i=0,1, \cdots, s,
$$

the given linear functionals $\omega_{i}$ being such that every polynomial $\phi(z)$ of degree $n \geqq 2 s$ for which

$$
\omega_{i}(\phi)=\sum_{k=0}^{2 s} \gamma_{k} c_{k}^{(i)}=0, \quad(i=0,1, \cdots, s), \quad \phi(z)=\sum_{k=0}^{n} \gamma_{k} z^{k},
$$

has $s+1$ roots at least in the domain $|z|<1$.

In the particular case when

$$
\omega_{i}(\phi)=\phi^{(i)}\left(z_{k}\right), \quad\left|z_{k}\right|<1,
$$

this lemma has been proved by M. S. Kakeya [1];1 without being aware of his result we have proved this lemma in the case ${ }^{2}$

\footnotetext{
${ }^{1}$ Numbers in brackets refer to the bibliography at the end. lems.

${ }^{2}$ In [1] and [2] one may find the application of this lemma to some extremal prob-
} 


$$
\omega_{i}(\phi)=\frac{1}{i !}\left(\frac{d^{i} \phi}{d z^{i}}\right)_{z=0}, \quad i=0,1, \cdots, s .
$$

In order to prove this lemma in the most general case we consider the following extremal problem:

Problem. To find the minimum of the integral

$$
L(b)=\int_{0}^{2 \pi}|t(z)|^{2} b(\theta) d \theta, \quad z=e^{i \theta},
$$

$t(z)$ being the given polynomial of degree $s$ with $t(0) \neq 0$ and $b(\theta)$ being a trigonometric polynomial of order $n \geqq 2 s$ :

$$
b(\theta)=R\left\{z^{n} \bar{\phi}\left(\frac{1}{z}\right)\right\}=R \sum_{k=0}^{n} \bar{\gamma}_{k} e^{i(n-k) \theta}, \quad z=e^{i \theta},
$$

subject to the conditions ${ }^{3}$

$$
\omega_{i}(b)=\omega_{i}(\phi)=\sum_{k=0}^{2 s} \gamma_{k} c_{k}^{(i)}=d_{i}, \quad i=0,1, \cdots, \mathrm{s} .
$$

The fundamental property of our functionals $\omega_{i}$ yields at once that every trigonometric polynomial $b(\theta)$ subject to the conditions

$$
\omega_{i}(b)=0, \quad i=0,1, \cdots, s,
$$

has in $(0,2 \pi)$ no more than $2(n-s-1)$ changes of sign. It is clear that there exists a solution of our problem. Further, the necessary conditions for an extremum are

$$
\operatorname{sgn} b^{*}(\theta)|t(z)|^{2}=R \sum_{k=n-2 s}^{\infty} A_{k} z^{k}, \quad z=e^{i \theta},
$$

whence we find at once that the Fourier expansion of $\operatorname{sgn} b^{*}(\theta)$ is of the form

$$
\operatorname{sgn} b^{*}(\theta)=R \sum_{k=n-s}^{\infty} B_{k} z^{k}, \quad z=e^{i \theta} .
$$

We have shown in [2] that every trigonometric polynomial with this property must be of the form

$$
b^{*}(\theta)=R\left\{\bar{c} z^{n-2 s+\nu} q^{2}(z)\right\} \tau(z) \bar{\tau}(1 / z), \quad z=e^{i \theta},
$$

$q(z)$ being a polynomial of degree $\sigma \leqq s$ all of whose roots lie in the domain $|z|<1$, and $\tau(z)$ being a polynomial of degree $\nu=s-\sigma$.

${ }^{3}$ The functionals $\omega_{i}$ are the same as above. 
The polynomial $b^{*}(\theta)$ for which the minimum is attained is unique. If there were two such polynomials, $b_{1}^{*}(\theta)$ and $b_{2}^{*}(\theta)$, then we would have

$$
L\left(b_{1}^{*}\right) \leqq L\left(\frac{b_{1}^{*}+b_{2}^{*}}{2}\right) \leqq L\left(b_{1}^{*}\right) ;
$$

then $b_{1}^{*}(\theta)$ and $b_{2}^{*}(\theta)$ would change sign at the same points, that is, the polynomial

$$
b_{1}^{*}(\theta)-b_{2}^{*}(\theta)=R\left\{z^{n-2 s+v} q^{2}(z)\right\}\left\{\bar{c}_{1}\left|\tau_{1}(z)\right|^{2}-\bar{c}_{2}\left|\tau_{2}(z)\right|^{2}\right\}, \quad z=e^{i \theta},
$$

would have at least $2(n-\nu)$ changes of sign in $(0,2 \pi)$; but since

$$
\omega_{i}\left(b_{1}^{*}-b_{2}^{*}\right)=0, \quad i=0,1, \cdots, s,
$$

the polynomial $b_{1}^{*}(\theta)-b_{2}^{*}(\theta)$ cannot have more than $2(n-s-1)$ changes of $\operatorname{sign}$ in $(0,2 \pi)$; this contradiction proves the unicity of the polynomial solving our problem. Thus we find that there exists the unique polynomial $b^{*}(\theta)$ minimizing (1) under conditions (2) and it must be of the form

$$
\begin{aligned}
b^{*}(\theta) & =R\left\{\bar{c} z^{n-2 s+v} q^{2}(z) \tau(z) \bar{\tau}(1 / z)\right\} \\
& =R\left\{\bar{\gamma}_{0}^{*} z^{n}+\bar{\gamma}_{1}^{*} z^{n-1}+\cdots+\bar{\gamma}_{2 s}^{*} z^{n-2 s}\right\}, \quad z=e^{i \theta} .
\end{aligned}
$$

Since the real parts of two polynomials coincide on the unit circle, these polynomials are identical, that is,

$$
\bar{c} z^{n-2 s} q^{2}(z) \tau(z) \tau^{*}(z)=\bar{\gamma}_{0}^{*} z^{n}+\bar{\gamma}_{1}^{*} z^{n-1}+\cdots+\bar{\gamma}_{2 s}^{*} z^{n-2 s},
$$

whence we find finally

$$
\phi^{*}(z)=\gamma_{0}^{*}+\gamma_{1}^{*} z+\cdots+\gamma_{2 s}^{*} z^{2 s}=c i^{2}(z) \tau(z) \tau^{*}(z),
$$

where

$$
i(z)=q^{*}(z)=z^{\sigma} \bar{q}(1 / z) .
$$

Thus we have found the polynomial $\phi^{*}(z)$ satisfying all the conditions of our lemma.

\section{BIBLIOGRAPHY}

1. S. Kakeya, Maximum modulus of some expressions of limited analytic functions, Transactions of this Society, vol. 22 (1921), pp. 489-504.

2. J. Geronimus, On a problem of F. Riesz and on the generalized problem of Tchebycheff-Korkine-Zolotareff, Bulletin de l'Académie des Sciences de l'URSS., vol. 3 (1939), pp. 279-288.

TECHNOLOGICAL Institute, KHARKOW, U.S.S.R. 